\title{
Enhanced Plasmonic Resonance Energy Transfer in Mesoporous Silica-Encased Gold Nanorod for Two-Photon-Activated Photodynamic Therapy
}

\author{
Nai-Tzu Chen 1,2, Kuo-Chun Tang'2, Ming-Fang Chung1, Shih-Hsun Cheng1, Ching-Mao Huang', Chia-Hui \\ $\mathrm{Chu}^{1}$, Pi-Tai Chou ${ }^{2}$, Jeffrey S. Souris ${ }^{3}$, Chin-Tu Chen ${ }^{3}$, Chung-Yuan Mou ${ }^{2}$, Leu-Wei Lo ${ }^{1}$ \\ 1. Institute of Biomedical Engineering and Nanomedicine (I-BEN), National Health Research Institutes, Zhunan, Miaoli 350, Taiwan. \\ 2. Department of Chemistry, National Taiwan University, Taipei 106, Taiwan. \\ 3. Department of Radiology, The University of Chicago, Chicago IL, 60637, USA.
}

\begin{abstract}
$\triangle$ Corresponding author: Leu-Wei Lo, Institute of Biomedical Engineering and Nanomedicine (I-BEN), National Health Research Institutes, Zhunan, Miaoli 350, Taiwan, Phone: +886-37-246-166 ext. 37115, Fax: +886-37-586-440. E-mail: lwlo@nhri.org.tw; or Chung-Yuan Mou, Department of Chemistry, National Taiwan University, Taipei 106, Taiwan, Phone: +886-2-3366-8205, Fax: +886-2-3366-8670. E-mail: cymou@ntu.edu.tw.
\end{abstract}

(C) Ivyspring International Publisher. This is an open-access article distributed under the terms of the Creative Commons License (http:/ / creativecommons.org/ licenses/by-nc-nd/3.0/). Reproduction is permitted for personal, noncommercial use, provided that the article is in whole, unmodified, and properly cited.

Received: 2014.02.25; Accepted: 2014.04.17; Published: 2014.05.30

\begin{abstract}
The unique optical properties of gold nanorods (GNRs) have recently drawn considerable interest from those working in in vivo biomolecular sensing and bioimaging. Especially appealing in these applications is the plasmon-enhanced photoluminescence of GNRs induced by two-photon excitation at infrared wavelengths, owing to the significant penetration depth of infrared light in tissue. Unfortunately, many studies have also shown that often the intensity of pulsed coherent irradiation of GNRs needed results in irreversible deformation of GNRs, greatly reducing their two-photon luminescence (TPL) emission intensity. In this work we report the design, synthesis, and evaluation of mesoporous silica-encased gold nanorods (MS-GNRs) that incorporate photosensitizers (PSs) for two-photon-activated photodynamic therapy (TPA-PDT). The PSs, doped into the nano-channels of the mesoporous silica shell, can be efficiently excited via intra-particle plasmonic resonance energy transfer from the encased two-photon excited gold nanorod and further generates cytotoxic singlet oxygen for cancer eradication. In addition, due to the mechanical support provided by encapsulating mesoporous silica matrix against thermal deformation, the two-photon luminescence stability of GNRs was significantly improved; after 100 seconds of $800 \mathrm{~nm}$ repetitive laser pulse with the 30 times higher than average power for imaging acquisition, MS-GNR luminescence intensity exhibited $\sim 260 \%$ better resistance to deformation than that of the uncoated gold nanorods. These results strongly suggest that MS-GNRs with embedded PSs might provide a promising photodynamic therapy for the treatment of deeply situated cancers via plasmonic resonance energy transfer.
\end{abstract}

Key words: Gold nanorods, photodynamic therapy, plasmonic resonance energy transfer, surface plasmon resonance, two-photon luminescence.

\section{Introduction}

Gold nanomaterials, and gold nanorods (GNRs) in particular, have recently received significant attention due to their photophysical and photochemical properties [1-3]. GNRs possess transverse and longitudinal surface plasmon resonances (SPRs) that cor- respond to electron oscillations perpendicular and parallel to the direction of the nanorod's major axis, respectively. The SPR band can be easily tuned from visible to infrared wavelengths through adjustments of the nanorod's aspect ratio. In addition, this unique 
SPR property contributes a local field enhancement of the radiative rate of electron-hole recombination processes, resulting in a considerable enhancement of the photoluminescence. The photoluminescence from GNRs is of particular interest in the fields of nanoscience and nanotechnology for various potential applications, including biomolecular sensing and imaging [4-8]. Photoluminescence from bulk gold was first reported by Mooradian in 1969 [9]. Subsequently, the mechanism and phenomenon was correlated with the electronic structure of gold nanoclusters, elucidated by Apell and Boyd $[10,11]$. Excitation takes place via transition of electrons from the d- to the sp-band, to generate electron-hole pairs. The observed emission results from the radiative electron-hole recombination of the excited electrons in the sp-band with the holes in the d-band, with SPR enhancement [11-13]. The luminescence of GNR is resonantly enhanced by a factor of approximately $10^{6}$ compared with that of bulk gold, a phenomenon known as the lightning-rod effect [14-16]. Heat is also generated as a consequence of electron-phonon collisions, garnered as the source for photothermal therapy.

Two-photon luminescence (TPL) from GNRs has considerable potential in biomedical imaging because of its high resolution, low photo-damage, good biocompatibility and low attenuation by water and biomaterials, enhancing photon penetration depth in tissue [17]. Although the quantum yield of emission of GNR is quite small, the fact that GNRs possess very high two-photon absorption cross-sections, about two order of magnitude higher than that of normal organic fluorophores [18], compensates enough to make GNR attractive as a two-photon luminescence imaging agent. For example, Chen et al. investigated a polystyrenesulfonate-coated GNR for two-photon photoluminescence imaging and in situ real-time photothermolysis in cancer cells [19]. Wang et al. also studied the in vitro and in vivo luminescence of GNRs in mouse ear blood vessels by two-photon activation and determined that the TPL intensity from a single GNR was 58 times brighter than that of a single rhodamine molecule [18]. For the application of photodynamic therapy (PDT), Yeh et al. coated poly(styrene-altmaleic acid) and indocyanine green onto the surface of GNRs, to form a nanoplatform for NIR optical imaging and photo-thermal/photodynamic therapy [20]. GNRs have also been used as a carrier and fluorescence quencher for PSs [21-23]. PSs have been conjugated onto the surface of GNRs via a protease-cleavable peptide linkage. Tumor-selective PDT and photothermolysis therapy (PTT) were performed after tumor targeting [22]. Photo-thermal effects generated by GNRs have also been exploited for externally controlled drug release via NIR lasers. Zhang et al. coated mesoporous silica on the outside of GNRs that served as multifunctional theranostic platforms: (a) the mesoporous $\mathrm{SiO}_{2}$ shell could provide a high surface area for the drug payload, while (b) the inner gold nanorod functioned as a two-photon imaging agent, hyperthermia agent, and a light-mediated trigger for drug release [24]. Same material with different formation, gold nanocages (AuNCs), was also employed for combination of two-photon photo-thermal/ photodynamic cancer therapy. The assembly of photosensitizer, hypocrellin B and photo-thermal transducer, AuNC with utilization of two-photon techniques results in one-off administration and irradiation for antitumor treatment. With NIR two-photon irradiation, AuNCs can convert light into heat and excite PS to generate singlet oxygen species [25].

However, studies have shown that repeated irradiation of GNRs and gold nanocrystals with intense laser pulses causes the GNRs to deform into gold nanospheres. Typically, pulse energies on the order of only $10 \mu \mathrm{J}$ are sufficient to completely convert gold nanorods into gold nanospheres [26-29]. The deformation of GNRs into spheroids inflicts a collateral spectral shift of peak surface plasmon resonance absorption, as well as a decrease in TPL intensity. To combat such losses, several groups have employed silica shell encasements of their nanorods as stabilizers to preclude thermal deformation of GNRs [30-32]. In previous work, we enveloped GNRs in a silica-matrix to enhance their stability during photoacoustic wave induction [30]. Our silica-coated GNRs demonstrated significantly greater resistance to degradation of signal intensity than that of the uncoated GNRs, significantly improving their use as high-contrast agents for photoacoustic imaging.

In this work, we report on the development of mesoporous silica-coated gold nanorods (MS-GNRs) bearing PSs, forming a stable and functionalizable probe for TPA-PDT. The mesoporous $\mathrm{SiO}_{2}$ shell deposited around our GNRs not only provided a large surface area for conjugating therapeutic moieties, but also served as a platform for intra-particle/plasmonic energy transfer between the GNRs and the payload materials. Providing confinement by mesoporous silica, the deformation of GNRs was significantly suppressed, with their TPL stability being greatly improved. For therapeutic application, not like widely used photothermal therapy, we incorporated a PS (Pd-meso-tetra(4-carboxyphenyl) porphyrin, PdTPP) into the nano-channels of the GNR's mesoporous silica shell. PdTPP is a metallo-porphyrin that not only could be used to measure oxygen distributions in tissues via oxygen-dependent quenching of phospho- 
rescence but also used as an efficient PS [33]. By conjugating PdTPP with MS-GNR, this nanoplateform can be made to switch between being a phosphorescence probe for oxygen sensing/imaging (diagnostics) and a PS for PDT (therapeutics) by simply changing the energy of photoirradiation. It opened the possibility of on-site diagnostic and therapeutic application in the future. Highly efficient excitation of the PS was achieved by proximal energy transfer from the GNRs surface plasmons to the PSs and then to neighboring oxygen molecules via collision. Following two-photon excitation of GNRs, cytotoxic singlet oxygen was generated and verified both in vitro and in vivo in a breast-cancer mouse models. As TPA-PDT employs near-infrared light, our PSs functionalized MS-GNRs achieve much greater penetration depths than PDT methods, making them well suited especially for the PDT treatment of deeper internal organs.

\section{Experimental Section}

Materials. Hexadecyltrimethylammoniumbromid (CTAB, 99\%, Acrôs organics), gold(III) chloride trihydrate $\left(\mathrm{HAuCl}_{4} \cdot 3 \mathrm{H}_{2} \mathrm{O}\right.$, sigma-Aldrich), tetraethyl orthosilicate (TEOS, 99.0\%, Aldrich), N,N-dimethylformamide (DMF, 99.8\%, Sigma-Aldrich), L-ascorbic acid (AA, 99.7\%, sigma-Aldrich), Sodium borohydride $\left(\mathrm{NaBH}_{4}, 99.9 \%\right.$, Fluka), Silver nitrate solution $\left(\mathrm{AgNO}_{3}, 0.01 \mathrm{~mol} / \mathrm{l}\right.$, Fluka), N-octane (98\%, Sigma-Aldrich), di(Nsuccinimidyl) carbonate (DSC, 95\% Fluka), $\mathrm{N}, \mathrm{N}$-diisopropylethylamine (99.5\%, Sigma-Aldrich), Deionized water $(18 \mathrm{M} \Omega$ ) was used in all the experiments.

Synthesis of MS-GNR. Gold nanorods were synthesized by seed-mediated growth according to previously described procedures [34]. First, $5 \mathrm{~mL}$ of CTAB solution $(0.20 \mathrm{M})$ was mixed with $5 \mathrm{~mL}$ of $\mathrm{HAuCl}_{4}$ solution $(0.5 \mathrm{mM})$. Subsequently, $0.60 \mathrm{~mL}$ of ice-cold $\mathrm{NaBH}_{4}$ solution $(0.01 \mathrm{M})$ was added to the mixture and vigorously stirred for 2 minutes at $28^{\circ} \mathrm{C}$, which resulted in the formation of a brownish-yellow seed solution. The growth solution was prepared by adding $0.2 \mathrm{~mL}$ of $\mathrm{AgNO}_{3}(4 \mathrm{mM})$ and $5 \mathrm{~mL}$ of $\mathrm{HAuCl}_{4}$ $(1 \mathrm{mM})$ solutions to $5 \mathrm{~mL}$ of CTAB $(0.20 \mathrm{M})$ solution under gentle mixing followed by the addition of $70 \mu \mathrm{L}$ of L-ascorbic acid $(0.0788 \mathrm{M})$ solution. To grow the nanorods, $12 \mu \mathrm{L}$ of the seed solution was added to the growth solution at $27-30{ }^{\circ} \mathrm{C}$ under gentle stirring for 30 seconds. The color of the solution changed to burgundy-red within 10-20 minutes. The solution was then aged for an additional 24 hours at $28{ }^{\circ} \mathrm{C}$ before being centrifuged at $12,000 \mathrm{rpm}$ for 30 minutes. For large-pore mesoporous silica coatings, $333 \mu \mathrm{L}$ n-octane and $0.10 \mathrm{~mL}$ of $0.1 \mathrm{M} \mathrm{NaOH}$ solution were added to $10 \mathrm{~mL}$ of the prepared GNR solution under stirring. Subsequently, $60 \mu \mathrm{L}$ of $20 \%$ TEOS in methanol was injected four times into the mixture at 30-minute intervals. The mixture was then allowed to react for 16 hours and was continued for an additional 4 hours after $35 \mu \mathrm{L}$ of APTMS (95\%) was added [35]. The resulting MS-GNR particles were then centrifuged and washed with DI water and ethanol at least three times to remove the CTAB. The surfactant templates were removed via the reaction with $0.1 \mathrm{M} \mathrm{HCl}$ for 20 hours followed by three washes with ethanol.

Synthesis of MS-GNR-PdTPP. We covalently linked the sensitizer group PdTPP to the surface of mesoporous silica by our previously reported method [36]. $40 \mathrm{mg}$ of Pd-meso-tetra(4-carboxyphenyl) porphyrin (PdTPP) and $150 \mathrm{mg}$ of DSC were mixed with $97 \mu \mathrm{L}$ of 1-Ethyl-3-(3-dimethylaminopropyl)carbodiimide in DMF $(20 \mathrm{~mL})$ solution for 2 hours. The activated PdTPP was then reacted with $100 \mu \mathrm{L}$ of APMTS for 1 hour and subsequently purified via passage through a PD-10 column (Amersham Biosciences). MS-GNR particles $(20 \mathrm{mg})$ were mixed with $400 \mu \mathrm{L}$ of APTMS-modified PdTPP in ethanol solution, and the mixture was stirred continuously at room temperature for 20 hours. Solid samples were collected via centrifugation at $12,000 \mathrm{rpm}$ for 20 minutes; the samples were subsequently washed, and the pellet was redispersed with deionized water and ethanol several times. The amount of PdTPP loading in the MS-GNRs was determined by measuring the absorbance of the reaction solution at $400 \mathrm{~nm}$ (i.e., the Soret band of PdTPP).

Two-photon luminescence and photostability measurements. The two-photon luminescence and photostability measurements were performed using an inverted scanning confocal microscope (Zeiss LSM 710). A femtosecond Ti:Sapphire laser (MaiTai, Spectra-Physics) was used as the excitation source to generate the TPL from MS-GNR. The output laser pulse was centered at $800 \mathrm{~nm}$, and the pulse duration was $\sim 200$ femtoseconds with a repetition rate of $80 \mathrm{MHz}$. The laser beam was directed into the microscope using a water-immersion objective lens $(\mathrm{N} . \mathrm{A}=1.4)$ to focus the laser beam onto the MS-GNRs, which were dispersed onto a clean glass coverslip with water. The TPL from MS-GNR was collected through the same objective, separated from the excitation laser by a dichroic beamsplitter, and then detected with a photomultiplier tube (PMT) placed in the back port of the microscope. A bandpass filter (400 to $690 \mathrm{~nm}$ ) was placed before the PMT to minimize the scattering of photons from the excitation source. For the photostability measurements, bare GNR or MS-GNR particles were irradiated under repetitive laser pulses with average power of $120 \mathrm{~mW}$ and the TPL was monitored as a function of time. For two-photon lumines- 
cence imaging, cells were monitored with excitation power of $4 \mathrm{~mW}$ after treatment of the MS-GNRs or MS-GNR-PdTPPs in a serum-free medium for 1 hour at $37^{\circ} \mathrm{C}$.

Singlet oxygen measurements. The chemical oxidation of 9,10-anthracendipropionic acid (ADPA) in an aqueous suspension of the nanoparticles was used to determine the singlet oxygen generation [37]. Aqueous solutions of ADPA $(20 \mu \mathrm{L}, 12.2 \mathrm{mM})$ were mixed with MS-GNR-PdTPPs solution in $\mathrm{D}_{2} \mathrm{O}(0.5 \mathrm{ml})$, whereas GNR solution with ADPA or PdTPP solution with ADPA was used as the control. All solutions were then irradiated under femtosecond laser with same power of $350 \mathrm{~mW}$ at $800 \mathrm{~nm}$ (Spitfire Pro, Spectra-Physics). The change in the absorbance of the ADPA at $378 \mathrm{~nm}$ was monitored as a function of time. All experiments were carried out in the dark room during the measurement.

In vitro two-photon luminescence images and PDT effects. MDA-MB-231 breast cancer cells were cultured in RPMI1640 medium containing $10 \%$ fetal bovine serum (FBS) at $37^{\circ} \mathrm{C}$ under $5 \% \mathrm{CO}_{2}$. Cells were seeded at a density of $3 \times 10^{4}$ on a $35 \mathrm{~mm}$ dish and incubated for 24 hours. To obtain two-photon luminescence images, the cells were treated with $50 \mu \mathrm{g} / \mathrm{mL}$ MS-GNR-PdTPPs in a serum-free medium for 1 hour at $37{ }^{\circ} \mathrm{C}$ in darkness and then washed twice with phosphate buffered saline (PBS). The cell nuclei were stained with $1 \mu \mathrm{M}$ Hoechst 33342 for 10 minutes and washed three times with PBS. To determine the in vitro PDT effects, $50 \mu \mathrm{g} / \mathrm{mL}$ MS-GNRs or MS-GNRPdTPPs were added to the cell dish with serum-free medium for 1 hour. Cells were washed with PBS to remove the excess nanoparticles and placed in fresh medium that contained YOPRO-1 and propidium iodide (PI) dyes (Molecular Probes, USA). GNRs were excited using a Ti:sapphire laser (Mai Tai, Spectra-Physics) at $800 \mathrm{~nm}$ with an output power of $3 \mathrm{~mW}$. For cell morphology and fluorescence imaging, YOPRO- 1 was excited at $488 \mathrm{~nm}$ and monitored using a $525 \pm 5 \mathrm{~nm}$ bandpass filter while PI was excited at 535 $\mathrm{nm}$ and monitored using a $620 \pm 5 \mathrm{~nm}$ bandpass filter. Images were collected using a confocal microscope (Zeiss LSM 710).

In vivo two-photon excited PDT effect. All experiments that involved animals were performed in accordance with the guidelines of the Management Group of Animal Experiments (MGAE) in Taiwan (NHRI-IACUC-101079-A). Male nude mice (nu/nu; 20-25 g; 6-8 weeks of age; BioLasco Taiwan) were anesthetized by isoflurane inhalation and subcutaneously inoculated with MDA-MB-231 cells $\left(2 \times 10^{6}\right.$ cells in $0.1 \mathrm{~mL}$ PBS). In vivo two-photon excited PDT experiments were performed when the tumor volume reached approximately $200 \mathrm{~mm}^{3}$. The nude mice were anaesthetized with $80 \mathrm{mg} / \mathrm{kg}$ ketamine and $13 \mathrm{mg} / \mathrm{kg}$ xylazine i.p. and received intra-tumor injections of sonicated MS-GNR-PdTPPs at a concentration of 16 $\mathrm{mg} / \mathrm{kg}$ in $100 \mu \mathrm{L}$ of saline solution. Control groups of mice received intra-tumor injections of saline, MS-GNRs or PdTPP alone. At 1 hour post-injection, the tumors in mice were irradiated using a Ti:Sapphire femtosecond amplifier (Spitfire Pro, Spectra-Physics). The femtosecond laser amplifier output was $1.2 \mathrm{~W}$ with a repetition rate of $1 \mathrm{kHz}$, pulse duration of $100 \mathrm{fs}$, central wavelength of $800 \mathrm{~nm}$, collimated beam diameter of $\sim 7 \mathrm{~mm}$ and Gaussian beam profile. An optical chopper (SR541, Stanford Research Systems) was used to block the laser and reduce the number of laser pulses onto the mice to $1 / 12$ to prevent direct photothermal heating damage to mice skin tissue. An average power of $100 \mathrm{~mW}$ was measured and irradiated onto the mice with exposure time of 16 minutes. The total exposure energy is $96 \mathrm{~J}$. For the histological evaluation, tumors were sectioned for hematoxylin and eosin, DAPI, TUNEL and caspase-3 staining. The slices were examined using an Olympus BX51 microscope.

Transmission electron microscopy (TEM). TEM imaging of GNR and MS-GNR particles was performed using a Libra 120 TEM transmission electron microscope (Carl Zeiss). A carbon-coated 200 mesh copper grid was pre-rinsed with $5 \mu \mathrm{l}$ of ethanol and then deposited with $5 \mu \mathrm{L}$ of the suspension of GNR or MS-GNR particles in ethanol. After 3 minutes, the sample was dried with filter paper and further dried with a flow of $\mathrm{N}_{2}$ gas. The sample was then viewed on the electron microscope, and images were obtained using a digital camera attached to the microscope. For TEM imaging of tumor slides, mice were sacrificed at 24 hours after MS-GNR-PdTPP treatment, and their tumor specimens were fixed overnight in glutaraldehyde buffered $(2.5 \%)$ with phosphate buffered saline (PBS; 0.1 M, pH 7.4). Tissues were then washed three times in PBS and post-fixed for 1 hour in a solution that contained $\mathrm{OsO}_{4}$ buffered (2\%) with PBS. Tissues were subsequently washed 3 times in $\mathrm{H}_{2} \mathrm{O}$ and dehydrated stepwise in ethanol. Tissues were then polymerized using Spurr resin at $68^{\circ} \mathrm{C}$ for 15 hours and embedded specimens subsequently sectioned into 70 nm slices and viewed on a Hitachi H-7650 TEM operated at $80 \mathrm{kV}$.

\section{Results and Discussion}

\section{Enhancement of the photostability of GNRs by the mesoporous silica confinement}

A description of the two-photon-activated photodynamic therapy using mesoporous-silica-encased 
gold nanorods (MS-GNR-PdTPP) is shown in Scheme 1.

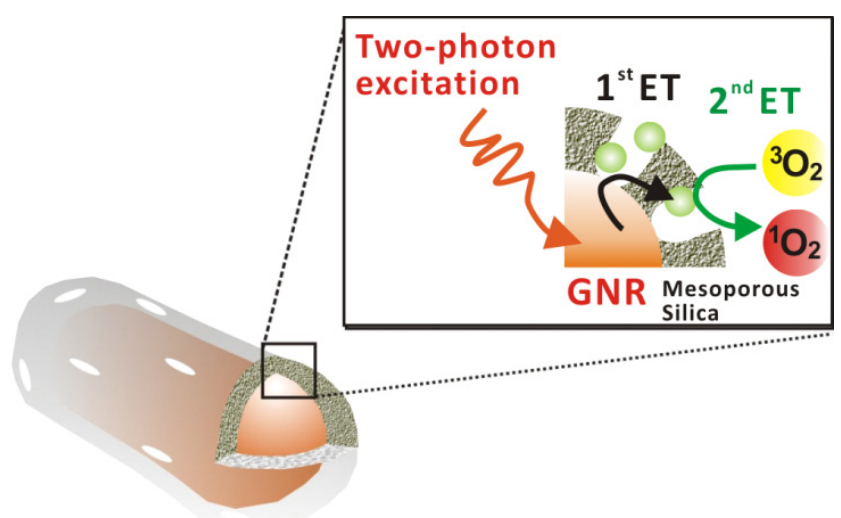

Scheme I. Schematic illustration of two-photon-activated photodynamic therapy (TPA-PDT) using mesoporous silica-encased gold nanorods. Pd-porphyrins (Green sphere), conjugated onto the walls of nanochannels within the mesoporous silica shell, are activated via intra-particle plasmonic energy transfer (ET) from two-photon-excited gold nanorods, to generate cytotoxic singlet oxygen.

In this approach, gold nanorods were synthesized via a seed-mediated, surfactant-assisted growth procedure [34], followed by its coating with mesoporous silica in a single step [35]. Pd-porphyrins were conjugated to the walls of nano-channels within the mesoporous silica shell via covalent bonds according to our previous method [36]. Following two-photon excitation, two sequential energy transfers were used to generate cytotoxic singlet oxygen: the first energy transfer ( $1^{\text {st }}$ ET), from the gold nanorod's surface plasmon to a proximal Pd-porphyrin within the silica shell, and the second energy transfer ( $2^{\text {nd }}$ ET) from the Pd-porphyrin to proximal oxygen molecules. Figure 1a shows TEM images of the MS-GNR with an average particle size of approximately $80 \mathrm{~nm}$. The average aspect ratio (length/diameter) of the gold nanorods was $3.4 \pm 0.80$. The inset of Figure 1a shows an enlarged image in which the mesoporous structure of the shell is clearly observed. The thickness of the mesoporous silica layer was less than $20 \mathrm{~nm}$. With a femtosecond pulsed laser beam directed into the microscope and onto a clean glass coverslip with water-dispersed MS-GNRs, photoluminescence measurements from the fixed scan area $(175 \mu \mathrm{m} \times 175 \mu \mathrm{m})$ were made. As shown in Figure 1b, a typical photoluminescence spectrum of an aqueous suspension of MS-GNRs excited at $800 \mathrm{~nm}$ via a femtosecond laser reveals a broad emission band in the visible region of $450-650 \mathrm{~nm}$. The oscillation structure in this range has been previously observed by Wang et al. [18] Boyd et al. had assigned them as transitions between the Fermi level and the various $d$ bands at various symmetry points [11]. Two-photon luminescence of MS-GNRs was confirmed by the non-linear dependence of the photoluminescence intensity on the exci- tation power. TPL measurements demonstrated a quadratic dependence of $1.98 \pm 0.30$ on the incident power, which confirmed that the excitation occurred via a two-photon absorption process (Figure 1c). Figure $1 \mathrm{~d}$ illustrates the relationship between the excitation spectrum of the two-photon luminescence (open triangles) and the longitudinal plasmon band (solid line) of the MS-GNRs. The significant overlap between the two spectra indicates that the observed two-photon luminescence intensity is governed by the local field enhancement originating from the gold nanorod's surface plasmon resonance. Evidently, MS-GNRs exhibit optical properties that are quite similar to those of bare GNRs.
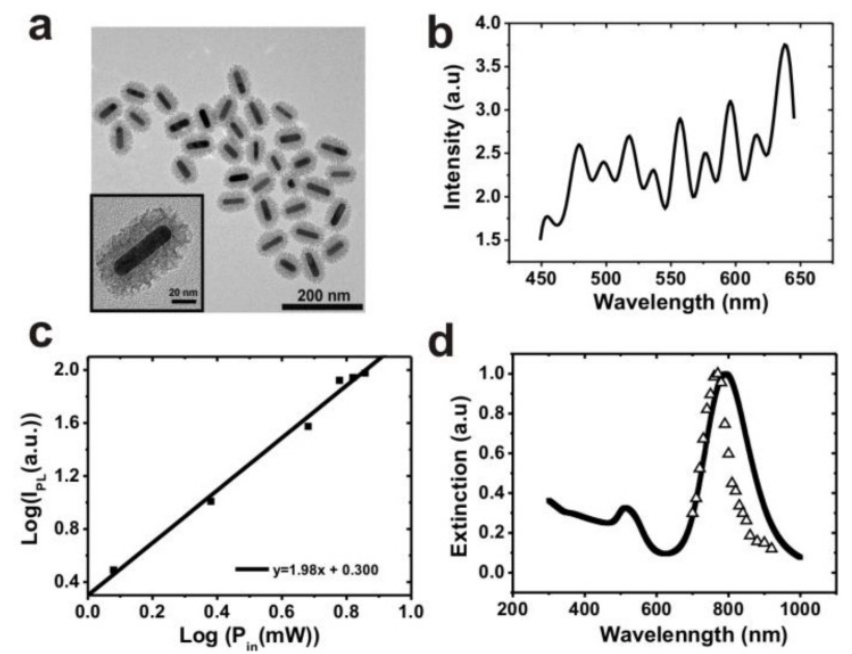

Figure I. TEM image and photophysical properties of MS-GNRs. (a) TEM image of MS-GNRs, with insert showing an enlarged view of its mesoporous structure. (b) Photoluminescence spectrum of MS-GNRs in an aqueous solution irradiated with femtosecond laser at $800 \mathrm{~nm}$. (c) Dependence of the integrated photoluminescence intensity of (b), versus the excitation power; slope, obtained from the regression fitting, is $\sim 2$ indicating that the measured photoluminescence is indeed TPL. (d) Steady-state absorption of MS-GNRs (solid line) and the TPL excitation spectrum (open triangles).

In Figure 2, we illustrate the robust stability of the two-photon luminescence intensity of MS-GNRs under repeated laser excitation, as compared with that of un-coated GNRs. Notably, although a small amount of residual CTAB remained on the surfaces of un-coated GNRs during the seed-mediated GNR synthesis, CTAB did not provide nearly as much protection of the nanorods from thermal deformation as did the mesoporous silica shell. Aqueous suspensions of GNR or MS-GNR particles were dispersed on clean glass coverslips, sealed by a rubber cassette to prevent dehydration and any consequent change in nanoparticle concentration. The average of two-photon luminescence intensities in a fixed regions-of-interest (ROIs) was measured and plotted as a function of time. The two-photon luminescence of uncoated GNRs (Figure 2a, black line) decayed rapidly to $39.4 \%$ of its initial value during the first 20 se- 
conds of exposure to $120 \mathrm{~mW}$ laser irradiation, and decreased further to $32.8 \%$ of its initial value after 40 seconds. In contrast, under identical laser irradiation conditions, the TPL of MS-GNRs (Figure 2a, red line) decayed to $94 \%$ of its initial maximal intensity during the first 20 seconds and remained relatively unchanged thereafter; with the MS-GNRs' TPL intensity being $260 \%$ greater than that of uncoated GNRs. After 100 seconds, the integrated TPL intensity of MS-GNRs was $220 \%$ greater than that of the uncoated GNRs.

TEM images of Figures $2 b$ and $2 c$ show the morphology of MS-GNRs, and GNRs respectively, after 100 seconds of laser irradiation. GNRs with a mean aspect ratio of $3.4 \pm 0.8$ before laser irradiation absorbed strongly at the $800-\mathrm{nm}$ excitation wavelength and underwent sufficient heating to undergo a shape transformation of many of the nanoparticles. The gentle surface melting of the GNRs leads to shorter, wider rods that possessed an average aspect ratio of 2.7 \pm 1.0 . Some of them have even melted into spherical shape (Figure 2c). In contrast, the shape of the nanorod cores within MS-GNRs possessed an average aspect ratio of $3.3 \pm 0.7$ after laser irradiation (Figure 2b). Thus, post-irradiated MS-GNRs retained most of the structural features of pre-irradiated MS-GNRs, consistent with the results of the stability of two-photon luminescence intensity measurements.

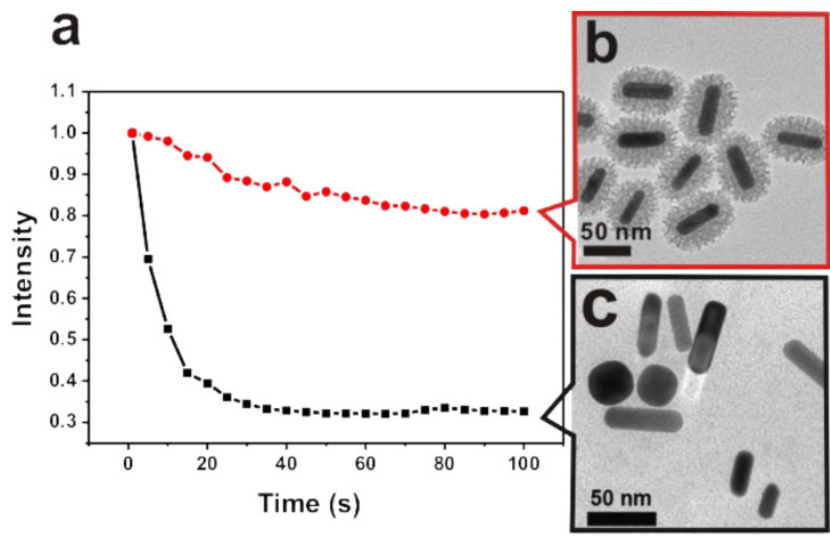

Figure 2. Enhancement of photostability of GNRs via mesoporous envelopment. (a) The TPL intensities of GNRs ( $\bullet$ ) and MS-GNRs (red $\bullet$ ) monitored as a function of time, under the exposure of $120 \mathrm{~mW}$ repetitive laser pulses. TEM images of MS-GNRs (b) and GNRs (c) following 100 seconds of laser irradiation.

\section{Photodynamic therapy by intra-particle plasmonic energy transfer}

Since the two-photon luminescence stability of gold nanorods is greatly enhanced by encapsulating the nanorods within a mesoporous silica matrix, we postulated that MS-GNRs could serve as sustained energy sources for PDT via a plasmonic energy transfer to the Pd-porphyrins adsorbed on the nearby silica shell under two-photon absorption. To substantiate this mechanism, we first examined the effects of conjugating Pd-porphyrins to MS-GNRs. In Figure 3a we present TEM images of MS-GNRs in which Pd-porphyrins had been conjugated onto the nano-channel walls of the mesoporous silica nanoparticles. As might be expected from the doping, the mesoporous structure of MS-GNR-PdTPPs in Figure $3 \mathrm{a}$ is darker than the structure of MS-GNR shown in Figure 1a. The surface charge of the MS-GNR-PdTPPs showed a positive $\xi$-potential value of $4.63 \mathrm{mV}$, indicating that the nanoparticles could be endocytosed well by MDA-MB-231 breast cancer cells (Figure 3c) [38]. Intra-particle plasmonic energy transfer was characterized by monitoring the emission spectrum within the visible region (450-650 nm) while exposed to 800-nm two-photon excitation. Luminescence of MS-GNRs was clearly noted within the visible spectrum (Figure 3b, black line) - advantageous for resonant energy transfer to the Pd-porphyrins. With the conjugation of Pd-porphyrins onto the nano-channel walls of MS-GNRs, the emission spectrum of MS-GNR-PdTPPs in Figure 3b (red line) showed a significant decrease at wavelengths $500-600 \mathrm{~nm}$, suggesting that highly efficient energy transfer had taken place between the gold nanorods and Pd-porphyrins following two-photon excitation. To evaluate the energy transfer of Pd-porphyrins to neighboring oxygen molecules, we monitored the generation of singlet oxygen via anthracene-9,10-dipropionic acid (ADPA), a singlet oxygen quencher which reacts with singlet oxygen and leads to reduction of its optical absorption at $378 \mathrm{~nm}$ [37]. Figure $3 \mathrm{~d}$ shows the decrease in optical absorption at $378 \mathrm{~nm}$ of an ADPA solution bearing MS-GNR-PdTPPs as a function of irradiation time reflecting efficient singlet oxygen production from the MS-GNR-PdTPP via 2-step serial energy transfers following two-photon excitation, with ADPA suspensions of PdTPP and GNRs serving as controls.

To characterize the cytotoxicity of two-photoninduced MS-GNR-PdTPP photodynamic therapy, two indicators cell damage were employed: YOPRO-1 and propidium iodide (PI). Because neither of these two dyes can penetrate cell membranes, counter-staining irradiated cells for YOPRO-1/PI signal reflected cell/nuclear membrane compromised. Repeated irradiation on MS-GNR-PdTPP-treated cells with a 800 $\mathrm{nm}$ femtosecond laser $(3 \mathrm{~mW})$ revealed increasing YOPRO-1 (green) and PI (red) penetration into cells as a function irradiation time, as shown in Figures 4a-c. Fluorescence intensity measurements of YOPRO-1 and PI, integrated and plotted as a function of time (Figure 4g), demonstrated that the fluorescence intensity of YOPRO-1 (green) and PI (red) increased by 18- and 94-fold respectively, following 194 seconds of laser irradiation. By contrast, no detectable change in 
the low levels of YOPRO-1 and PI fluorescence was observed for cells treated with MS-GNR (Figures 4d-f and $4 h)$.
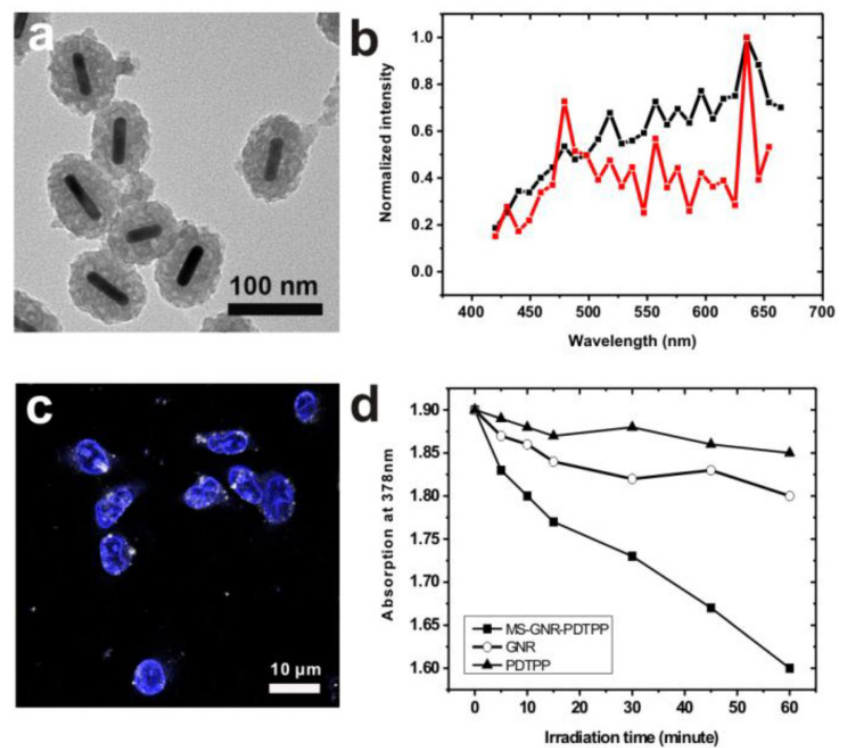

Figure 3. (a) TEM imaging of MS-GNR-PdTPPs; scale bar: $0.10 \mu \mathrm{m}$ (b) Energy transfer in MS-GNR-PdTPPs, showing the TPLs of MS-GNRs (black) and MS-GNR-PdTPPs (red). (c) Confocal microscopy imaging of the endocytosis of MS-GNR-PdTPPs, with the nuclei stained by Hochest 33342 (blue signal); white foci are the TPLs of MS-GNR-PdTPPs under $800 \mathrm{~nm}$ laser excitation; scale bar: $10 \mu \mathrm{m}$. (d) Decay of optical absorption of ADPA at $378 \mathrm{~nm}$, caused by the generation of singlet oxygen from GNRs (O), PDTPPs $(\boldsymbol{\Delta})$ and MS-GNR-PdTPPs $(\boldsymbol{\square})$ as a function of laser irradiation exposure time.
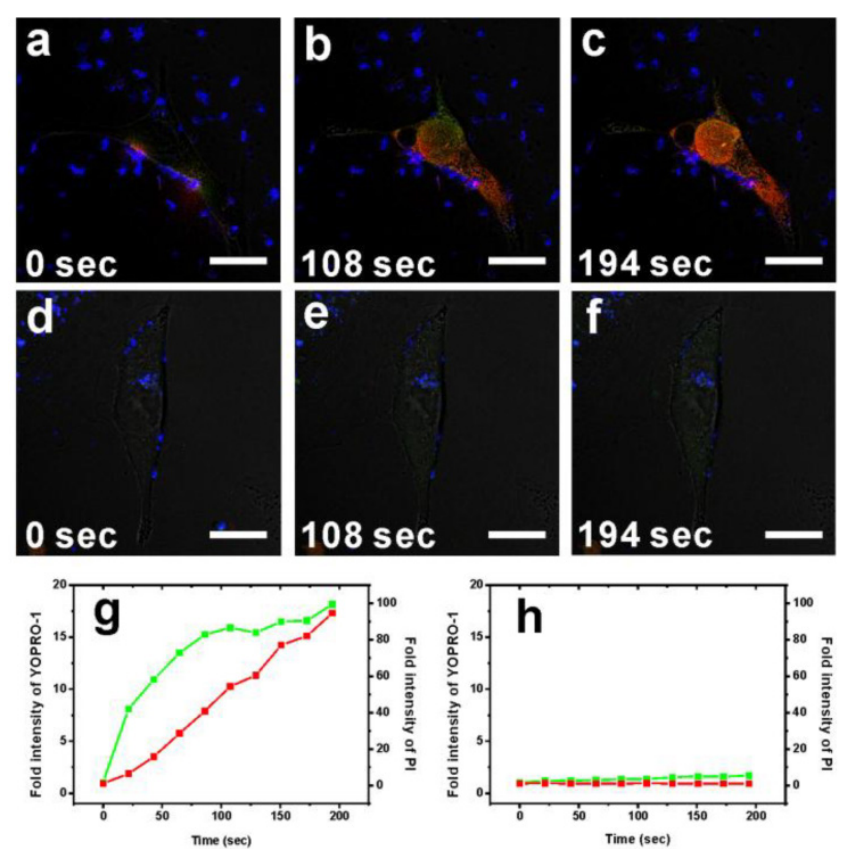

Figure 4. Confocal fluorescence microscopy images showing the TPA-PDT performance ( $\mathrm{O}_{2}$ cytotoxicity) of MS-GNR-PdTPPs (a)-(c) and MS-GNRs (d)-(f) in treated breast cancer cells under $3 \mathrm{~mW}$ repetitive laser irradiation of 3 durations: 0 , 108 , and 194 seconds. YOPRO-I (green) and propidium iodide signals (red) denote leakage of the cell and nuclear membranes, respectively. Scale bar: $10 \mu \mathrm{m}$. Fluorescence intensity of YOPRO-I and propidium iodide as a function of irradiation period for cells treated with MS-GNR-PdTPPs $(\mathrm{g})$ and MS-GNRs (h).

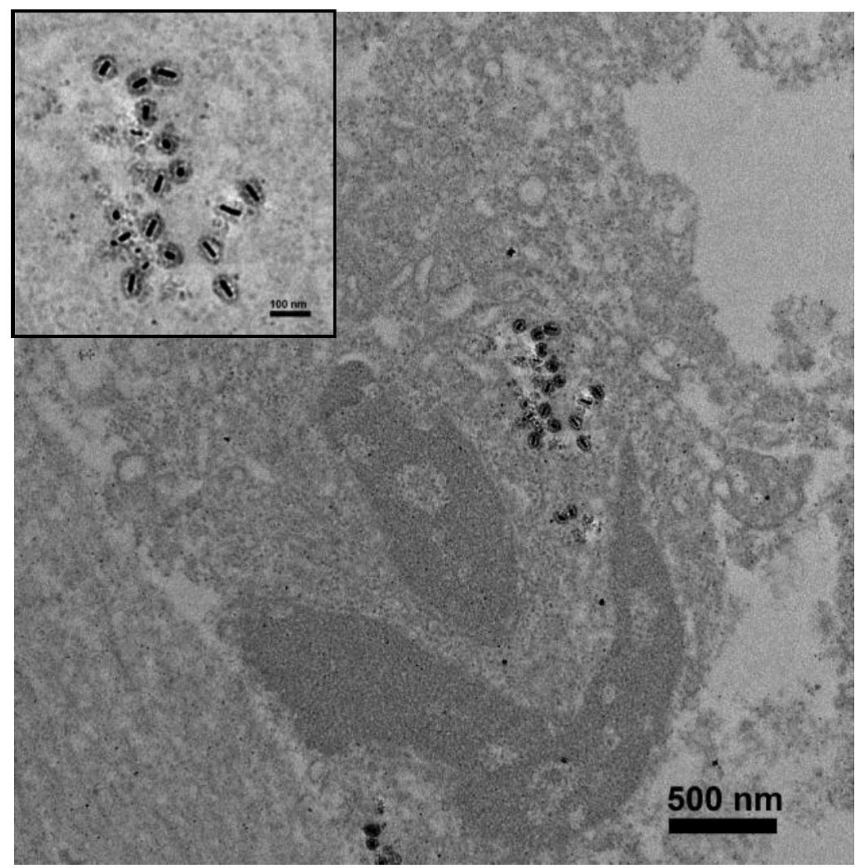

Figure 5. TEM images of a $70 \mathrm{~nm}$-thick section of tumor harvested from an anesthetized mouse 24 hours after administration of MS-GNR-PdTPPs and TPA-PDT; scale bar: $500 \mathrm{~nm}$. Insert shows an enlarged image of localized MS-GNR-PdTPPs within the tumor; scale bar: $100 \mathrm{~nm}$.

To evaluate the in vivo efficacy of two-photon-induced MS-GNR-PdTPP photodynamic therapy, nude mouse models were implanted with $2 \times$ $10^{6}$ MDA-MB-231 breast cancer cells and treated with $16 \mathrm{mg} / \mathrm{kg}$ of MS-GNR-PdTPPs by intra-tumor injection. In vivo localization of MS-GNR-PdTPPs was accomplished by TEM imaging of the tumor tissue from an anesthetized mouse 24 hours after injection of nanoparticles and subsequent irradiation. As Figure 5 shows, MS-GNRs-PdTPPs were endocytosed by cells without suffering any structural defects (see inset of Figure 5).

To assess in vivo TPA-PDT efficacy, a breast tumor xenograft mouse model was irradiated with 800 $\mathrm{nm}$ femtosecond laser pulses for a total exposure energy of $96 \mathrm{~J}(\sim 250 \mathrm{~J} / \mathrm{cm} 2), 1$ hour after injection of MS-GNR-PdTPP (Figure 6g-i) nanoparticles. An optical chopper was used in this system to reduce the delivered number of laser pulses to its $1 / 12$. It prevented the tissue damages caused by direct laser irradiation and the following induced GNR photothermal effect. By such configuration, the photothermal effects, not like in other studies [24-25], could be minimized to enable analysis of only the photodymanic phenomena of PS induced by the enhanced plasmonic energy transfer of GNR. The mouse was sacrificed 24 hours post-irradiation and its tumor harvested/sectioned into $5 \mathrm{~mm}$ thick slices. Tissue sections were then stained with one of two apoptosis indicators - Caspase-3 and TUNEL - to elucidate cy- 
totoxicity. Hematoxylin and eosin (H\&E) and DAPI stains were also applied to characterize each specimen's morphology. The results of the staining are shown in Figure 6 for tumors subject no treatment (i.e., the blank controls; Figures 6a-c), to irradiation alone (Figures 6e-g), treatment with MS-GNRs followed by irradiation (Figures 6m-o), and treatment with MS-GNR-PdTPPs but without irradiation (Figures 6q-s); for comparison to the results of TPA-PDT experiments (Figures 6i-k). Caspase-3 activity was assessed by caspase-3 immunohistochemistry with caspase- 3 antibodies and displayed by DyLight (ex. $654 \mathrm{~nm}$, em. $673 \mathrm{~nm}$ )-conjugated goat-anti-mouse secondary antibodies (Figure 6, third row, red). Apoptosis-induced DNA fragmentation was revealed by terminal deoxynucleotidyl transferase dUTP nick end labeling (TUNEL) (Figure 6, second row, green). As Figure 6 shows, none of the tumor tissues were stained by casepase- 3 and TUNEL except for those subjected to combined MS-GNR-PdTPP treatment and laser irradiation. Represented tumor images $(n=3$ for each group) acquired 14 days after treatments were shown for quantitative analysis of tumor growth (Figure 6, bottom row). Tumors grew 5.12 times (control, figure $6 \mathrm{~d}$ ), 4.62 times (laser, figure $6 \mathrm{~h}$ ), 4.2 times (MS-GNR-PdTPP, figure 6t) and 3.41 times (MS-GNR, figure $6 p$ ) compare to the original size on the first day. By contrast, inhibition of tumor growth was observed with combined MS-GNR-PdTPP treatment and laser irradiation (1.2 times, figure 6i). Despite relatively large pulse energies $(1.2 \mathrm{~mJ} /$ pulse $)$ employed, the optical chopping reduced exposure levels sufficiently as to permit heat dissipation - No apparent direct (Figures 6e-h) and limited indirect (Figures 6m-p) photothermal heating effects were observed under the laser irradiations used in this experiment. Thus simple, optically chopped femtosecond laser can be used to perform TPA-PDT at the relatively safe average power of $\sim 100 \mathrm{~mW}$ irradiated on a $7 \mathrm{~mm}$ circular area (i.e., $250 \mathrm{~mW} / \mathrm{cm} 2$ ), with minimal collateral damage to neighboring tissue.

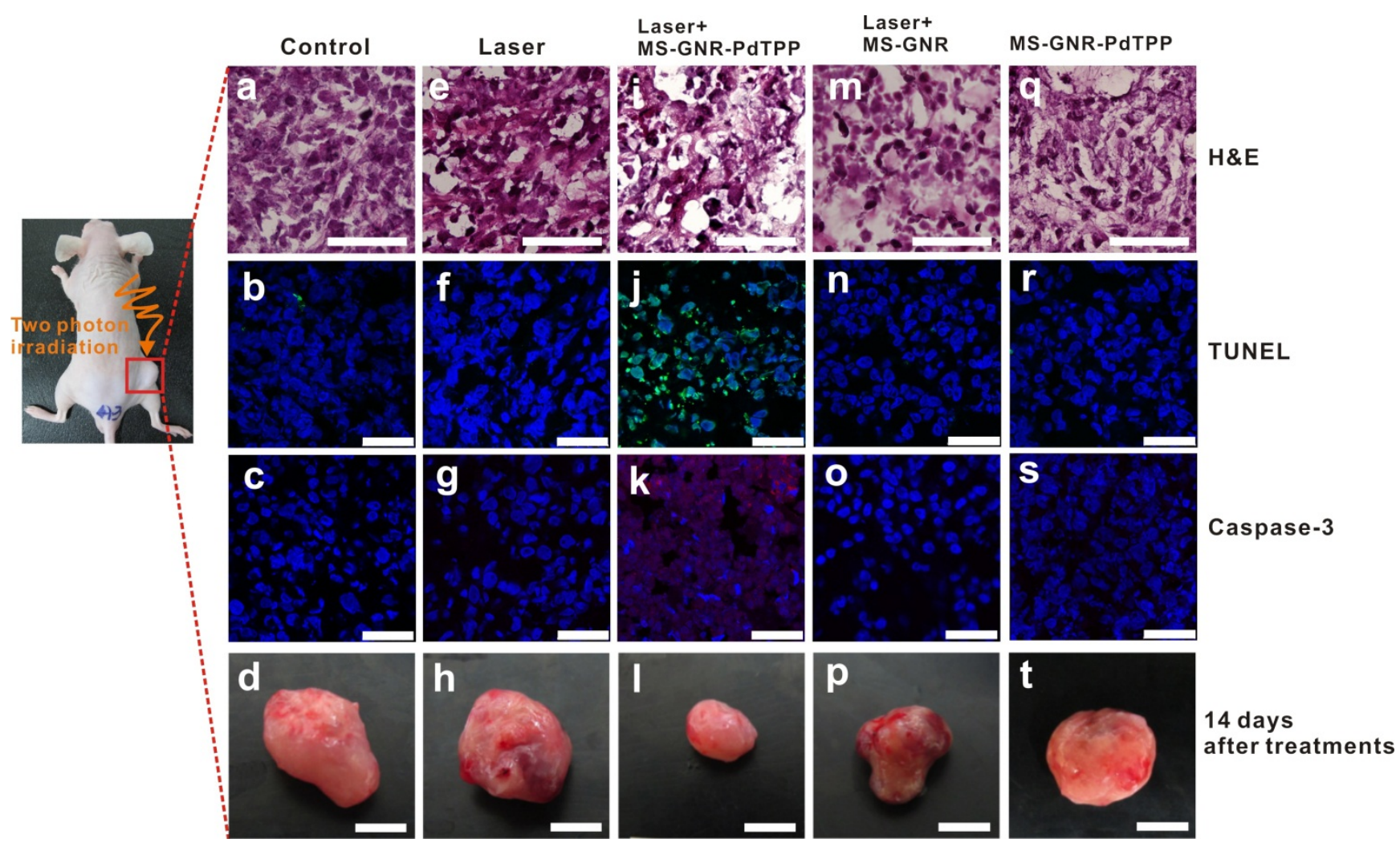

Figure 6. In vivo studies illustrating TPA-PDT therapeutic benefits derived from intra-tumor injection of MS-GNR-PdTPPs. Histologic analyses of harvest tumor sections 24 hours post-irradiation were made with hematoxylin and eosin (first row, scale car: $100 \mu \mathrm{m}$ ), TUNEL (second row, green coloring), caspase-3 immuno-histogram (third row, red coloring) and DAPI (blue coloring) staining. Scale bar: $25 \mu \mathrm{m}$. Represented tumor images ( $\mathrm{n}=3$ for each group) acquired I4 days after treatments were shown for quantitative analysis of tumor growth (fourth row). Scale bar: $5 \mathrm{~mm}$.

\section{Conclusion}

In this report, we describe the development of a novel intra-particle plasmonic energy transfer nanocomposite for two-photon photo-dynamic therapy comprised of mesoporous silica-encased gold nanorods. Enveloping the gold nanorods with mesoporous silica confers a number of advantages to the use of bare or non-mesoporous silica coated GNRs; these include: 
(1) Stabilization of the gold nanorods' shape against thermal deformation following repeated laser irradiation.

(2) Enhanced two-photon excited luminescence intensity and imaging lifetime of the nano-platform, which extended the possibility for deeper and longer imaging in vitro and in vivo.

(3) Large surface area for conjugation of photosensitizers.

(4) Scaffold for intra-particle plasmonic energy transfer between the GNRs and the photosensitizers preventing photosensitizers from GNR quenching by directing conjugation. It improves the subsequent energy transfer efficiency of photosensitizers to oxygen molecules for generation of cytotoxic singlet oxygen.

Using a 2-step sequential intra-particle energy transfer upon two-photon excitation (from gold nanorod $\rightarrow$ Pd-porphyrin $\rightarrow$ oxygen molecule,), we observed substantial cytotoxic sequelae in both in vitro cell studies and in vivo murine models of breast cancer.

Taken together, the intense two photon luminescence coupled with good stability of mesoporous silica encased gold nanorods, integrated with intra-particle energy transfer activated singlet oxygen generation, make mesoporous silica-encased gold nanorods a promising nanosystem for the efficient in vivo tracking and photodynamic therapy of malignancies in deep tissues.

\section{Abbreviations}

GNR: gold nanorod; MS-GNR: mesoporous silica-coated gold nanorod; GM: Units of Goeppert-Mayer; PDT: photodynamic therapy; TPA-PDT: two-photon activated-photodynamic therapy; TPL: two-photon luminescence; SPR: surface plasmon resonances.

\section{Acknowledgment}

The study was supported by the National Health Research Institutes of Taiwan (MED-099-PP-04 and NM-099-PP-01) and by the National Science Council of Taiwan (NSC 98-2221-E-400-001 and NSC 99-2113-M-400-001-MY3). We thank Chun-Wei Shih for performing the confocal microscopic image acquisition and Ms. Yu-Ching Chen for assistance with the TEM measurements.

\section{Competing Interests}

The authors have declared that no competing interest exists.

\section{References}

1. Xia Y, Li W, Cobley CM, Chen J, Xia X, Zhang Q, Yang M, Cho EC, Brown PK. Gold nanocages: from synthesis to theranostic applications. Acc Chem Res. 2011; 44: 914-924.

2. Alkilany AM, Thompson LB, Boulos SP, Sisco PN, Murphy CJ. Gold nanorods: Their potential for photothermal therapeutics and drug delivery, tempered by the complexity of their biological interactions. Adv Drug Deliv Rev. 2012; 64: 190-199.

3. Liu CL, Wu HT, Hsiao YH, Lai CW, Shih CW, Peng YK, Tang KC, Chang H W, Chien YC, Hsiao JK. Insulin-directed synthesis of fluorescent gold nanoclusters: preservation of insulin bioactivity and versatility in cell imaging. Angew Chem Int Edit. 2011; 50: 7056-7060.

4. Cheng JX, Tong L, Wei QS, Wei A. Gold nanorods as contrast agents for biological imaging: optical properties, surface conjugation and photothermal effects. Photochem and Photobiol. 2009; 85: 21-32.

5. Qian J, Zhan QQ, Li X, He SL. A study of mesoporous silica-encapsulated gold nanorods as enhanced light scattering probes for cancer cell imaging. Nanotechnology. 2010; 21 (5): 055704-055712.

6. Kuo WS, Chang CN, Chang YT, Yang MH, Chien YH, Chen SJ, Yeh CS. Gold nanorods in photodynamic therapy, as hyperthermia agents, and in near-infrared optical imaging. Angew Chem Int Edit. 2010; 49: 2711-2715.

7. Tong L, He W, Zhang Y, Zheng W, Cheng JX. Visualizing systemic clearance and cellular level biodistribution of gold nanorods by intrinsic two-photon luminescence. Langmuir. 2009; 25(21): 12454-12459.

8. Imura K, Okamoto H. Properties of photoluminescence from single gold nanorods induced by near-field two-photon excitation. J Phys Chem C. 2009; 113: 11756-11759.

9. Mooradian, A. Photoluminescence of Metals. Phys Rev Lett. 1969; 22: 185-187.

10. Apell P, Monreal R, Lundqvist S. Photoluminescence of noble metals. Phys Scr. 1988; 38: 174-179.

11. Boyd GT, Yu ZH, Shen YR. Photoinduced luminescence from the noble metals and its enhancement on roughened surfaces. Phys Rev B Condens Matter. 1986; 33: 7923-7936.

12. Wang, D. S.; Hsu, F. Y.; Lin, C. W. Surface plasmon effects on two photon luminescence of gold nanorods. Opt Express. 2009; 19: 11350-11358.

13. Lin A, Son DH, Ahn IH, Song GH, Han WT. Visible to infrared photoluminiscence from gold nanoparticles embedded in germano-silicate glass fiber. Opt express. 2007; 15: 6374-6379.

14. Mohamed MB, Volkov V, Link S, El-Sayed MA. The 'lightning' gold nanorods: fluorescence enhancement of over a million compared to the gold metal. Chem Phys Lett. 2000; 317: 517-523.

15. Yorulmaz M, Khatua S, Zijlstra P, Gaiduk A, Orrit M. Luminescence quantum yield of single gold nanorods. Nano Lett. 2012; 12: 4385-4391.

16. Eustis S, El-Sayed MA. Why gold nanoparticles are more precious than pretty gold: Noble metal surface plasmon resonance and its enhancement of the radiative and nonradiative properties of nanocrystals of different shapes. Chem Soc Rev. 2006; 35: 209-17.

17. Durr NJ, Larson T, Smith DK, Korgel BA, Sokolov K, Ben-Yakar A. Two-photon luminescence imaging of cancer cells using molecularly targeted gold nanorods. Nano Lett. 2007; 7: 941-945.

18. Wang H, Huff TB, Zweifel DA, He W, Low PS, Wei A, Cheng JX. In vitro and in vivo two-photon luminescence imaging of single gold nanorods. Proc Natl Acad Sci U S A. 2005; 102: 15752-6.

19. Chen CL, Kuo LR, Chang CL, Hwu YK, Huang CK, Lee SY, Chen K, Lin SJ, Huang JD, Chen YY. In situ real-time investigation of cancer cell photothermolysis mediated by excited gold nanorod surface plasmons. Biomaterials. 2010; 31: 4104-4112.

20. Yeh CS, Kuo WS, Chang CN, Chang YT. Antimicrobial gold nanorods with dual-modality photodynamic inactivation and hyperthermia. Chem Comm. 2009; 32: 4853-4855.

21. Jang B, Park JY, Tung CH, Kim IH, Choi Y. Gold nanorod-photosensitizer complex for near-infrared fluorescence imaging and photodynamic/photothermal therapy in vivo. ACS Nano. 2011; 5: 1086-1094.

22. Jang B, Choi Y. Photosensitizer-conjugated gold nanorods for enzyme-activatable fluorescence imaging and photodynamic therapy. Theranostics. 2012; 2: 190-7.

23. Zhao T, Wu H, Yao SQ, Xu QH, Xu GQ. Nanocomposites containing gold nanorods and porphyrin-doped mesoporous silica with dual capability of two-photon imaging and photosensitization. Langmuir. 2010; 26: 14937-14942.

24. Zhang Z, Wang L, Wang J, Jiang X, Li X, Hu Z, Ji Y, Wu X, Chen C. Mesoporous silica-coated gold nanorods as a light-mediated multifunctional theranostic platform for cancer treatment. Adv Mater. 2012; 24: 1418-23.

25. Gao L, Fei J, Zhao J, Li H, Cui Y, Li J. Hypocrellin-loaded gold nanocages with high two-photon efficiency for photothermal/photodynamic cancer therapy in vitro. ACS Nano. 2012; 6(9): 8030-8040.

26. Chen YS, Frey W, Kim S, Homan K, Kruizinga P, Sokolov K. Emelianov, S. Enhanced thermal stability of silica-coated gold nanorods for photoacoustic imaging and image-guided therapy. Opt Express. 2010; 18: 8867-8877.

27. Wu X, Yang F, Ming T, Xiong R, Wang P, Chen J. Au nanorods can be used for long-term cell imaging? Appl Phys Lett. 2011; 98: 213704.

28. Link S, El-Sayed MA. Shape and size dependence of radiative, non-radiative and photothermal properties of gold nanocrystals. Int Rev Phys Chem. 2000; 19: 409-453. 
29. Bouhelier A, Bachelot R, Lerondel G, Kostcheev S, Royer P, Wiederrecht GP. Surface plasmon characteristics of tunable photoluminescence in single gold nanorods. Phys Rev Lett. 2005; 95: 267405-267409.

30. Chen LC, Wei CW, Souris JS, Cheng SH, Chen CT, Yang CS, Li PC, Lo LW. Enhanced photoacoustic stability of gold nanorods by silica matrix confinement. J Biomed Optics. 2010; 15: 016010-016016.

31. $\mathrm{Wu} \mathrm{C}, \mathrm{Xu} \mathrm{OH}$. Stable and functionable mesoporous silica-coated gold nanorods as sensitive localized surface plasmon resonance (LSPR) nanosensors. Langmuir. 2009; 25: 9441-9446.

32. Chen YS, Frey W, Kim S, Kruizinga P, Homan K, Emelianov S. Silica-coated gold nanorods as photoacoustic signal nanoamplifiers. Nano Lett. 2011; 11: 348-354

33. Cheng SH, Lee CH, Yang CS, Tseng FG, Mou CY, Lo LW. Mesoporous silica nanoparticles functionalized with an oxygen-sensing probe for cell photodynamic therapy: potential cancer theranostics. J Mater Chem. 2009; 19: 1252-1257.

34. Nikoobakht B, El-Sayed MA. Preparation and growth mechanism of gold nanorods (NRs) using seed-mediated growth method. Chem Mater. 2003; 15 : 1957-1962.

35. Gorelikov I, Matsuura N. Single-step coating of mesoporous silica on cetyltrimethyl ammonium bromide-capped nanoparticles. Nano Lett. 2008; 8: 369-373.

36. Cheng SH, Lee CH, Chen MC, Souris JS, Tseng FG, Yang CS, Mou CY, Chen CT, Lo LW. Tri-functionalization of mesoporous silica nanoparticles for comprehensive cancer theranostics - the trio of imaging, targeting and therapy. J. Mater. Chem., 2010;20:6149-6157.

37. Hoebeke M, Piette J, ven de Vorst A. Photosensitized production of singlet oxygen by merocyanine 540 bound to liposomes. J Photochem Photobiol B. 1991; 9 (3-4): 281-294.

38. Chung TH, Wu SH, Yao M, Lu CW, Lin YS, Hung Y, Mou CY, Chen YC, Huang DM. The effect of surface charge on the uptake and biological function of mesoporous silica nanoparticles in 3T3-L1 cells and human mesenchymal stem cells. Biomaterials. 2007; 28: 2959-2966. 